\title{
Nanoelectromechanical nonvolatile memory device incorporating nanocrystalline Si dots
}

\author{
Yoshishige Tsuchiya, ${ }^{\text {a) }}$ Kosuke Takai, Nobuyuki Momo, Tasuku Nagami, Hiroshi Mizuta, \\ and Shunri Oda \\ Quantum Nanoelectronics Research Center and Department of Physical Electronics, Tokyo Institute \\ of Technology, and SORST, Japan Science Technology Agency (JST), 2-12-1 O-okayama, Meguro-ku, \\ Tokyo 152-8552, Japan \\ Shinya Yamaguchi and Toshikazu Shimada \\ Central Research Laboratory, Hitachi Ltd., 1-280, Higashi-Koigakubo, Kokubunji-Shi, Tokyo 185-8601, \\ Japan
}

(Received 10 April 2006; accepted 29 June 2006; published online 3 November 2006)

\begin{abstract}
A nanoelectromechanical device incorporating the nanocrystalline silicon (nc-Si) dots is proposed for use as a high-speed and nonvolatile memory. The nc-Si dots are embedded as charge storage in a mechanically bistable floating gate. Position of the floating gate can therefore be switched between two stable states by applying gate bias. Superior on-off characteristics are demonstrated by using an equivalent circuit model which takes account of the variable capacitance due to the mechanical displacement of the floating gate. Mechanical property analysis conducted by using the finite element method shows that introduction of nc-Si dot array into the movable floating gate results in reduction of switching power. High switching frequency over $1 \mathrm{GHz}$ is achieved by decreasing the length of the floating gate to the submicron regime. We also report on experimental observation of the mechanical bistability of the $\mathrm{SiO}_{2}$ beam fabricated by using the conventional silicon etching processes. (C) 2006 American Institute of Physics. [DOI: 10.1063/1.2360143]
\end{abstract}

\section{INTRODUCTION}

Various bistable natures observed for nonsilicon materials have been explored intensively for developing nonvolatile and random access memory (RAM) in order to achieve high operating speed and long data-retention time simultaneously. For example, ferroelectric RAM, magnetic RAM, and phase-change RAM are currently investigated as serious candidates. However, all those memory devices require introduction of an unconventional material into silicon device fabrication processes. An alternative bistability mechanism is therefore worth exploring only by using the conventional silicon based materials.

Application of silicon micromachining technologies to the nonvolatile memory device by using mechanical bistability of the object was proposed by Peterson ${ }^{1}$ and developed by Hälg. ${ }^{2}$ Silicon mechanical objects have a clear advantage of future integration with advanced silicon circuits because they can be fabricated with conventional silicon technology consisting of oxidation, thin film deposition, lithography, and etching. ${ }^{3}$ The operating speed of the electromechanical systems depends on the size of the mechanical object and it is generally slower than the electrical switching speed on the scale of micrometer range. However, significant progress of silicon nanofabrication techniques has also enabled to realize the silicon nanoelectromechanical systems (NEMS) with sizes of below $1 \mu \mathrm{m}$ along with the rapid scaling down of the metal-oxide-semiconductor field-effect transistor (MOSFET) device. Since the operation frequency of electromechanical systems increases with decreasing size, an ex-

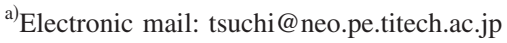

tremely high-speed operation is expected in the downsized NEMS. Recently, Huang et al. reported about $1 \mathrm{GHz}$ oscillation of the $\mathrm{SiC}$ beam, the dimension of which was $1.1 \mu \mathrm{m}$ long, $120 \mathrm{~nm}$ wide, and $75 \mathrm{~nm}$ thick. ${ }^{4}$ One order improvements were made in a decade for both of the beam size and the oscillation frequency. ${ }^{5}$ Such high-speed motion in the gigahertz range was also predicted on the multiwall carbon nanotube with the length of $100 \mathrm{~nm} .^{6,7}$

All these results could give us an idea that the bistable nature of the NEMS has the possibility to realize a highspeed nonvolatile memory device with the operation speed of several gigahertz or higher. In this paper, we discuss silicon nanoelectromechanical nonvolatile memory device incorporating nanocrystalline-Si (nc-Si) dots. We used nc-Si dots as charge storage in the mechanically movable object with bistable nature. The operation principle of the NEMS memory device in detail is described in the following section. The movable floating gate is a key building block of the NEMS memory device. We studied fundamental mechanical properties of the movable floating gate theoretically in Sec. III. We also mention about switching speed of the nanoscale movable floating gate estimated by transient analysis. Fabrication of a single beam structure and experimental observation of mechanical bistability of the beam is shown in Sec. IV. Section V is dedicated to the summary of this paper. Part of this work has already been reported elsewhere. ${ }^{8,9}$

\section{NONVOLATILE NANODOT NEMS MEMORY DEVICE}

Figure 1 shows a schematic cross-sectional view of the nanodot NEMS memory device. The NEMS memory features a suspended floating gate beam in the cavity placed 


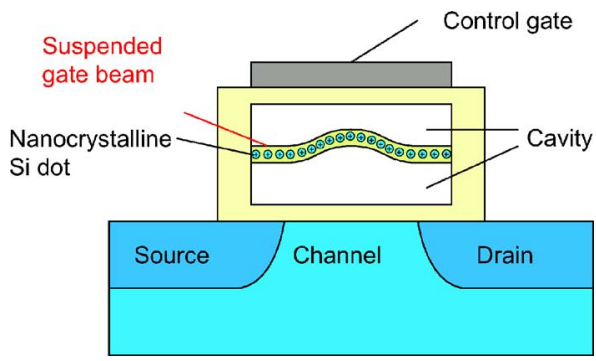

FIG. 1. (Color online) A schematic illustration of a NEMS memory device incorporating nanocrystalline-Si dots.

under the gate electrode, which contains the nc-Si dots as charge storage. The floating gate is bent naturally, either upward or downward. Its ends are clamped at the cavity side walls. When the gate voltage is applied, the floating gate beam moves via electrostatic interactions between the gate electrode and the charge in the nc-Si dots. A positional displacement of the floating gate may be sensed via a change in the drain current of the MOSFET underneath. Mechanical bistability of the beam is therefore essential for achieving our nonvolatile NEMS memory, and a higher switching speed is expected with reducing their dimensions down to the nanom- eter regime. While the conventional flash memory has big issues of tunnel oxide degradation caused by repeated charge tunneling and low write and erase speed, our NEMS memory device has the advantages of no charge tunneling and the high-speed write and erase operation. In addition, fabrication process of the NEMS is compatible with conventional $\mathrm{Si}$ process without introducing any other strange materials. We can estimate the on-off ratio of this device simply from the difference of surface potential change induced by applied gate bias between upward- and downward-bent beams. We assume the substrate is $p$-type $\mathrm{Si}$, and the positive charge is stored in the beam. The upward- and downward-bent states correspond to " 0 " or "off" state and " 1 " or "on" state, respectively. Structural parameters used for the present calculations are shown in Fig. 2(a), where the cavity height is $200 \mathrm{~nm}$ and the initial displacement of the beam is assumed to be $50 \mathrm{~nm}$. Figure 2(b) shows an equivalent circuit we use in this estimation. In Fig. 2(b), $C_{U}$ is the capacitance between the metal gate and the floating gate, $C_{L}$ is the capacitance between the floating gate and the substrate surface, and $Q$ is the charge stored and confined in the floating gate. Applied gate bias $V_{g}$ is given as (a)

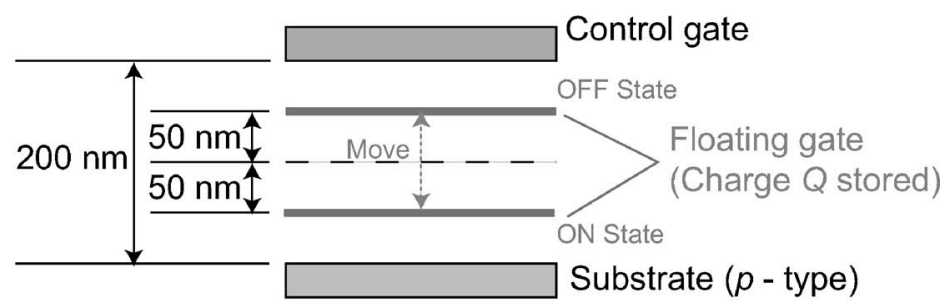

(b)

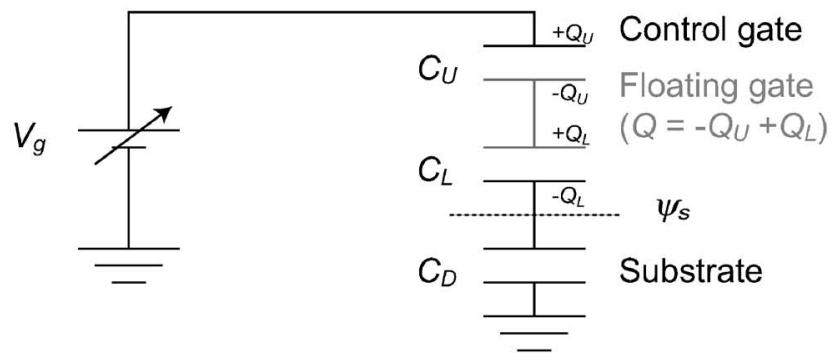

(c)

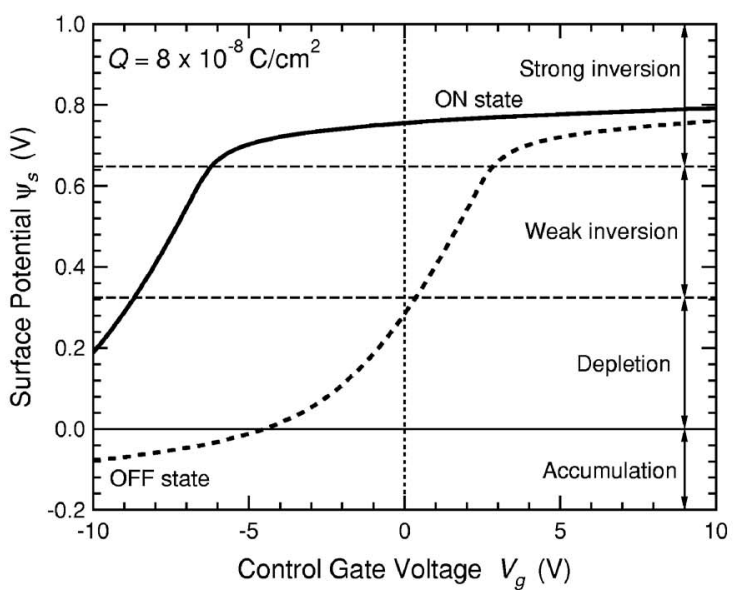

FIG. 2. (a) A schematic illustration of the NEMS memory cell model used in the on-off ratio calculation. The position of the beam in "On" and "Off" states is $50 \mathrm{~nm}$ lower or higher than the center between surfaces of control gate and substrate in the cavity. (b) Equivalent circuit of the NEMS memory cell. The dashed line shows the position of the surface of the substrate. $C_{D}$ is the capacitance of the depletion layer. (c) Calculated surface potential $\psi_{s}$ as a function of applied gate voltage $V_{g}$. The solid and dashed lines correspond to the On and Off states, respectively. Cases at the semiconductor surface in a certain $\psi_{s}$ range are depicted on the right hand side with arrows. 


$$
V_{g}=\frac{Q_{U}}{C_{U}}+\frac{Q_{L}}{C_{L}}+\psi_{s}
$$

where $Q_{U}$ and $Q_{L}$ are charges that emerge from the capacitor surfaces and $\psi_{s}$ is the surface potential on the silicon substrate. The stored charge,

$$
Q=-Q_{U}+Q_{L},
$$

is assumed to be maintained in the beam. The positional change of the floating gate induces the change in $C_{U}$ and $C_{L}$, and then varies $Q_{U}$ and $Q_{L} . Q_{L}$ is equal to the absolute value of the surface charge $\left|Q_{s}\right|$, which is related to $\psi_{s}$ as

$$
Q_{L}=\left|Q_{s}\right|=\frac{\sqrt{2} k T}{q L_{D}} F\left(\psi_{s}, \frac{n_{p 0}}{p_{p 0}}\right),
$$

where $L_{D}$ is the Debye length, and $n_{p 0}$ and $p_{p 0}$ are the electron and hole densities in the $p$-type substrate. ${ }^{10}$ Figure 2 (c) shows surface potential curves for the on and off states calculated from Eqs. (1)-(3) as a function of gate voltage. We found large on-off ratio of $10^{10}$ from the difference between $\psi_{s}$ 's of on and off states at $V_{g}=0$ in the case with $Q$ of 8 $\times 10^{-8} \mathrm{C} / \mathrm{cm}^{2}$, which corresponds to $n_{s}$ of $5 \times 10^{11} \mathrm{~cm}^{-2}$, where $n_{s}$ is the number density of charge storage in a unit area. Note that the on-off ratio at $V_{g}=0$ was reduced to 10 in the case with $Q$ of $1.6 \times 10^{-7} \mathrm{C} / \mathrm{cm}^{2}$, which corresponds to $n_{s}$ of $1.0 \times 10^{12} \mathrm{~cm}^{-2}$, and is not sufficient for switching devices. The above analysis shows that device operation is quite sensitive to the amount of charge in the beam. If we use a beam incorporating nc-Si of several nanometers in diameter, we can easily control the area density of the charge in the beam. Once an electron is stored in such a small Si nanodot, another electron transfer probability into the dot is strongly reduced due to the Coulomb blockade effect even at room temperature. ${ }^{11}$ The amount of charge in the beam can therefore be determined by the number of Si nanodots. We used a very-high-frequency digital plasma process for nc-Si deposition that facilitates deposition of nc-Si dots of $8 \pm 1 \mathrm{~nm}$ in diameter. ${ }^{12,13}$ Density of the $8 \mathrm{~nm}$ nc-Si dots is typically about $10^{11}-10^{12} \mathrm{~cm}^{-2}$ in a monolayer, which are the same order with $n_{s}$ required for the large on-off ratio operation. We can control the amount of the nc-Si dots precisely by adjusting the deposition condition, therefore, we believe that this technique is suitable for fabricating the charged beam.

\section{THEORETICAL STUDIES OF BASIC DEVICE CHARACTERISTICS}

In this section, we discuss the basic device characteristics theoretically. Mechanical properties of the movable floating gate beam are closely related to the fundamental characteristics of the memory operation, such as the switching speed and the operation voltage. We therefore estimate several basic device characteristics by using the finite element method (FEM) simulation, which is frequently used in the analysis of mechanical properties of materials in terms of elastic continuum approximation. (a)

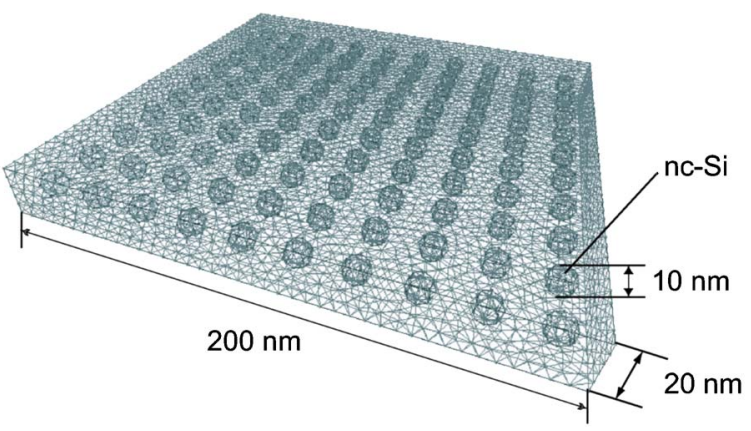

(b)

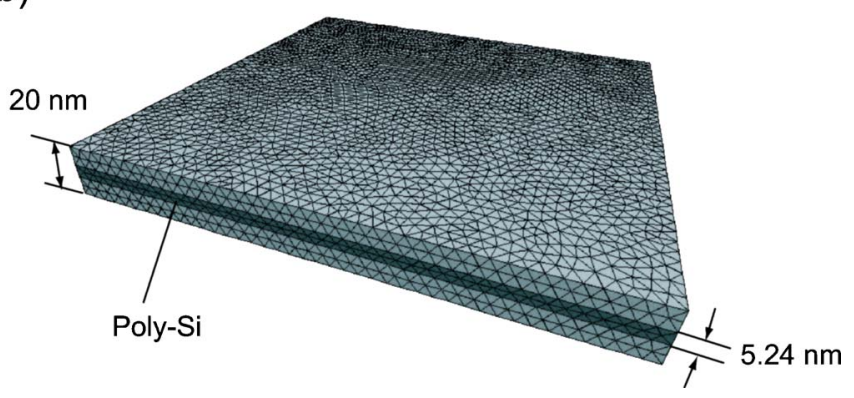

FIG. 3. (Color online) (a) A 3D image of a nc-Si beam model used in this calculation. The 2D array of spheres (nc-Si dots) is clearly identified in the sheet $\left(\mathrm{SiO}_{2}\right)$. (b) An image of a poly-Si beam model in which a thin sheet $(\mathrm{Si})$ is placed between two $\mathrm{SiO}_{2}$ layers. The fine lines in the figures show the boundary of the mesh used in this study.

\section{A. Effect of nc-Si incorporation on the mechanical properties}

First we study the effects of nc-Si incorporation on the mechanical properties of the movable beam. Mechanical properties of the beam were analyzed by using ADVENTURE system, ${ }^{14}$ a parallel three-dimensional (3D) FEM simulator. Here we focus on the nc-Si beam structure, in which a twodimensional array of nc-Si dots with the diameter of $10 \mathrm{~nm}$ is embedded in a $\mathrm{SiO}_{2}$ film [Fig. 3(a)]. We also investigated a simple poly-Si beam structure, in which a thin $\mathrm{Si}$ sheet of $5.24 \mathrm{~nm}$ in thickness is placed between $\mathrm{SiO}_{2}$ layers [Fig. $3(b)]$ as a reference. The size of both beams is $200 \times 200$ $\times 20 \mathrm{~nm}^{3}$, and the ratio of volume of $\mathrm{Si}$ to that of $\mathrm{SiO}_{2}$ is the same for both beams. Young's modulus and Poisson's ratio are $190 \mathrm{GPa}$ and 0.27 for $\mathrm{Si}$, and $70 \mathrm{GPa}$ and 0.175 for $\mathrm{SiO}_{2}$, respectively. ${ }^{15,16}$ Figure 4 shows a calculated 3D image of the beam deformed under a constant homogeneous pressure with $5.0 \times 10^{-10} \mathrm{~N} / \mathrm{nm}^{2}$ parallel to the $z$ axis, where the $z$ axis is perpendicular to the initial beam plane. We found the

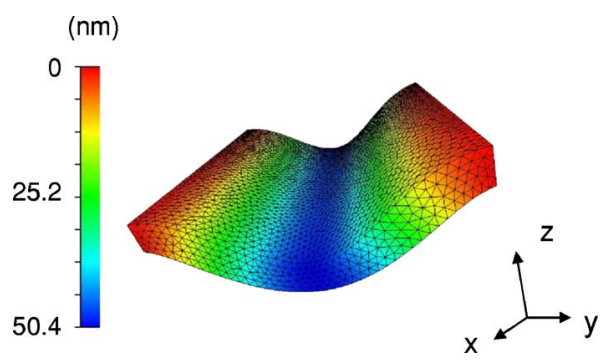

FIG. 4. (Color online) A calculated 3D image of the nc-Si beam model deformed under a constant homogeneous load parallel to the $z$ axis. Dark area around the center of the beam was largely deformed. 
maximum central displacement of $50.4 \mathrm{~nm}$ for the $\mathrm{nc}-\mathrm{Si}$-dot beam, which is larger than that of $48.5 \mathrm{~nm}$ for the poly-Si beam with the same pressure. The result suggests that the nc-Si-dot beam has an advantage for low power consumption, because the relatively larger displacement was obtained against the same applied force, which corresponds to the applied gate voltage in terms of the NEMS memory operation.

\section{B. Mechanical bistability}

Next, we study mechanical bistability of the beam by using NE/NASTRAN,${ }^{17}$ which takes account of the nonlinearity in the load-displacement relationship. The buckling of the beam should be introduced in the nonlinear analysis properly because it is an essential phenomenon to realize the bistability in the NEMS memory. Dimensions of the nc-Si-dot beam are $400 \mathrm{~nm}$ in length, $200 \mathrm{~nm}$ in width, and $20 \mathrm{~nm}$ in thickness. The initial buckled state was formed from the plane beam as follows. First we introduce the internal stress by loading a small force of $0.1 \mu \mathrm{N}$ along the $x$ axis parallel to the longest dimension of the beam. After we apply a light load along $-z$ direction, the initial downward-bent buckled state was formed with the initial displacement of about $24 \mathrm{~nm}$. With increasing the load along the $+z$ direction gradually, a sudden change of the displacement took place due to the buckling of the beam at a point where the load strength was about $0.5 \mu \mathrm{N}$. We also observed a similar sudden change during gradual loading along $-z$ direction. Figure 5 shows the maximum central displacement of the beam from $z=0$ as a function of applied force, together with beam shapes of the upward- and downward-bent states, respectively. The hysteresis observed in Fig. 5 is a direct evidence of bistability.

In estimation of elastic potential $U$, first we shall evaluate the restoring elastic force $f$ against the beam deformation as a function of the beam displacement. $U$ can be calculated from $f$ by the following integration; $U=-\int f d z$. Figure 6 displays $U$ as a function of the beam displacement. We can clearly identify a double minimum structure which is characteristic of the bistable system. Note that the height of the potential at zero displacement corresponds to the potential barrier of switching between two bistable states. The value is directly related to the magnitude of switching voltage. Although the current result shows rather higher value about $10 \mathrm{kV}$, we can reduce this bias voltage down to several tenth volts by choosing appropriate structural parameters of the beam-in-cavity memory cell. Details will be described elsewhere. ${ }^{18}$

\section{Estimation of write and erase operation speed}

In this section, we estimate the write and erase operation speed. Here, we have to consider the frequency of the oscillation between two bistable states, which corresponds to write and erase operation speed of the nonvolatile NEMS memory device. Note that this frequency is different from the resonance frequency of the beam. First, we briefly mention the result of a quite simple model based on the equation of motion, $m d^{2} Z / d t^{2}=F \mp f$, where $Z$ is the maximum displace-

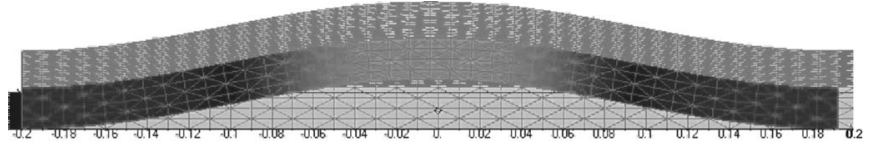

\section{Off State}
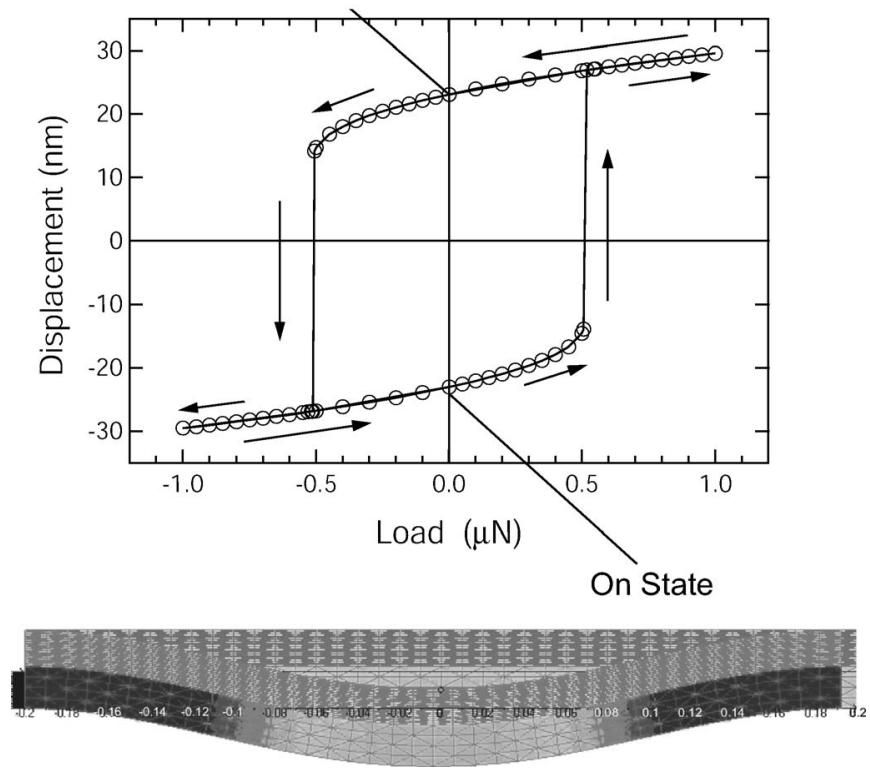

FIG. 5. A displacement of the beam was plotted as a function of applied load parallel to the $z$ axis. Abrupt change of the displacement of the beam and clear hysteresis were observed, which are due to the mechanical bistable nature of the beam. The images above and below the figure are the shape of upward-bent beam at the Off state and that of downward-bent beam at the On state, respectively.

ment of the beam, $m$ is the total mass of the beam, and $F$ is the external force applied to the beam along the $z$ axis. The elastic force $f$ is represented as $f=k S \Delta V / V$, where $k$ is the elastic volume constant, $S$ is the loading area, $V$ is the volume of the beam, and $\Delta V$ is the volume change of the deformed beam. If $Z$ is positive, where $f$ is acting against $F$, we use the minus sign in the equation of motion; if $Z$ is negative, the plus sign is used. We roughly estimated the oscillation frequencies of about $2 \mathrm{GHz}$ for the $\mathrm{SiO}_{2}$ beam with the size of $1 \times 1 \times 0.1 \mu \mathrm{m}^{3}$ and of about $20 \mathrm{GHz}$ with the size of

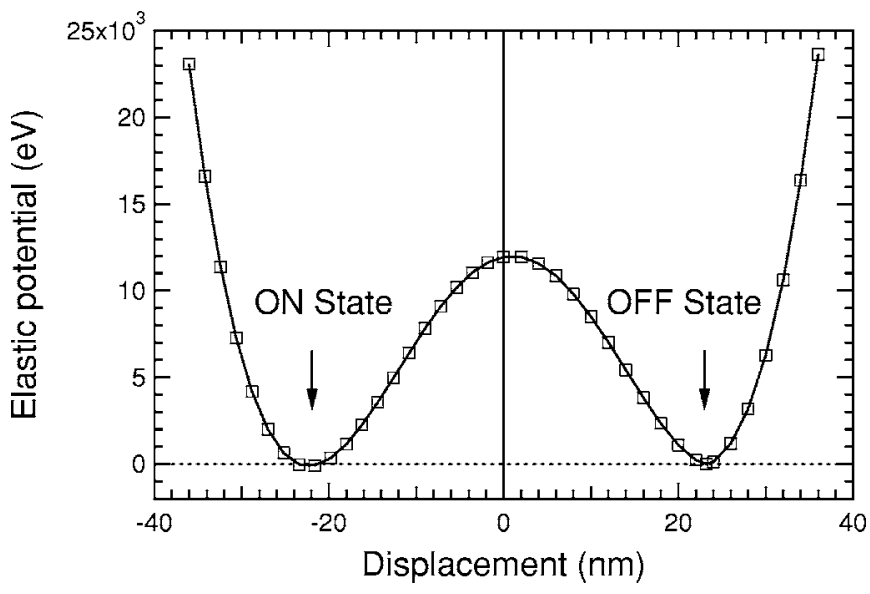

FIG. 6. Elastic potential as a function of the displacement of the beam. Double minimum structure which is characteristic of the system bistability is clearly observed. 


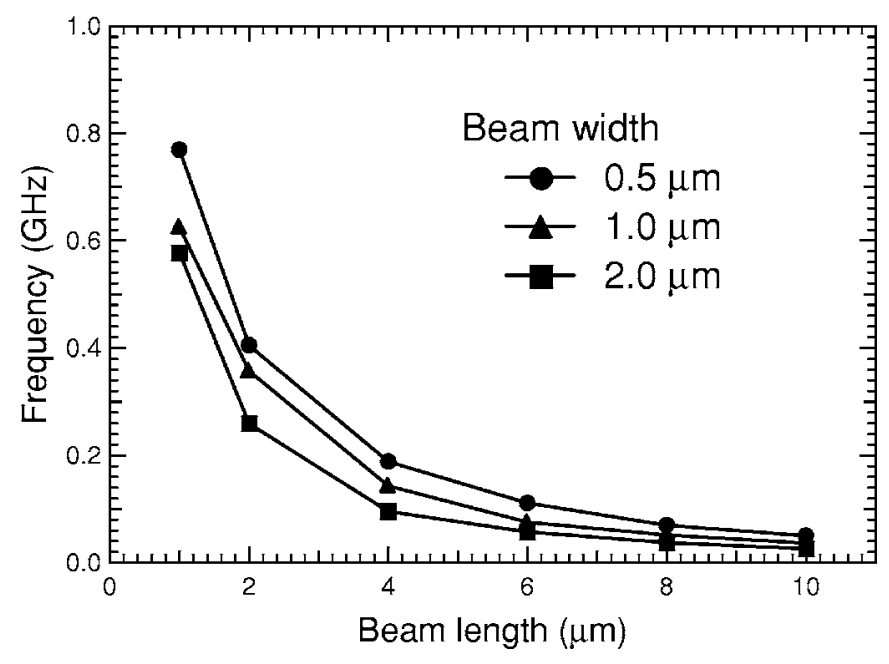

FIG. 7. The oscillation frequencies of the beam as a function of the beam length are shown for beams with three different widths; $0.5,1.0$, and $2.0 \mu \mathrm{m}$.

$0.1 \times 0.1 \times 0.01 \mu \mathrm{m}^{3}$ from this calculation. The speed of the write/erase operation in the NEMS memory was therefore more than $10^{3}$ times higher than that of the conventional current flash memory.

In order to investigate the size dependence of switching speed further, we used transient analysis algorithm in the nonlinear FEM simulator. ${ }^{17}$ The damping of the motion was ignored in this estimation. First we made a model shape of the beam with an initial displacement of $100 \mathrm{~nm}$. Then the concentrated load of $300 \mu \mathrm{N}$ was applied to the beam for $0.1 \mathrm{~s}$. An oscillation was clearly identified in the time evolution of the beam displacement. The oscillation frequency calculation was performed for various beams with different dimensions. Figure 7 shows the estimated oscillation frequency as a function of beam length at three different beamwidths. The frequency increases with decreasing beam length and beamwidth and increases rapidly below $4 \mu \mathrm{m}$ of the beam length. About $0.8 \mathrm{GHz}$ was achieved for the beam with the size of $1 \mu \mathrm{m}$ length and $0.5 \mu \mathrm{m}$ width. Furthermore, rapid increase up to gigahertz range is expected with further reduction of the beam length. These estimations show that the NEMS memory is promising for nonvolatile memory, where the high-speed write and erase operation is realized.

\section{EXPERIMENTAL STUDIES OF THE DEVICE FABRICATION}

In the following section, we show results of a single beam structure fabrication which is considered as a step towards the beam-in-cavity structure fabrication. The single beam structure fabrication is also suitable for observing the basic mechanical properties of the beam with a size below several micrometers. We also show the result of nanoindentor test for the single beam structure.

\section{A. Fabrication of single beam structure}

Schematic illustration of the single beam structure is shown in Fig. 8(a), and the fabrication process is as follows.
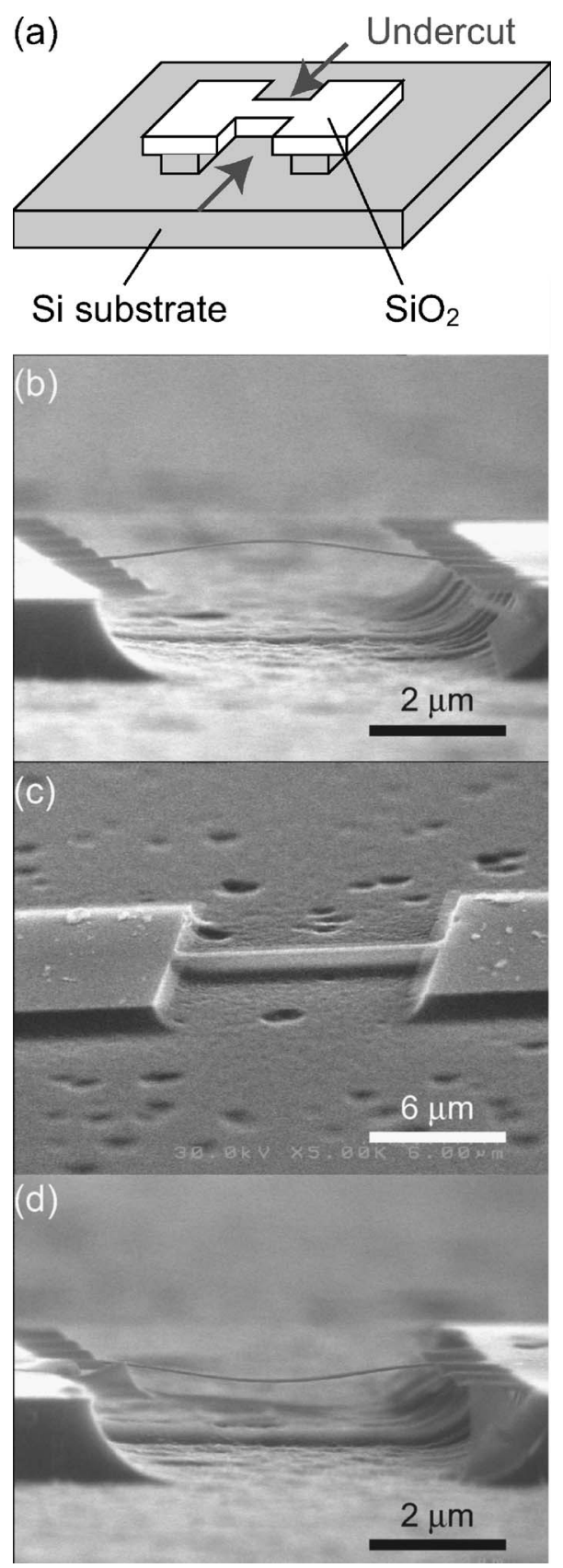

FIG. 8. (a) A schematic illustration of a $\mathrm{SiO}_{2}$ single layer structure fabricated by etching of $\mathrm{Si}$ underneath using $\mathrm{CF}_{4} / \mathrm{O}_{2}$ plasma. The SEM images of (b) an upward-bent thermally grown $\mathrm{SiO}_{2}$ beam, (c) a PECVD-grown $\mathrm{SiO}_{2}$ beam with no bending, and (d) a downward-bent thermally grown $\mathrm{SiO}_{2}$ beam. Only thermally grown $\mathrm{SiO}_{2}$ beams show bending after undercutting.

After wafer cleaning, 170-nm-thick $\mathrm{SiO}_{2}$ was formed by dry oxidation followed by patterning with the help of photolithography or electron-beam lithography. The pattern was copied to $\mathrm{SiO}_{2}$ by using anisotropic dry etching with $\mathrm{CF}_{4}$ gas. A $2 \mu \mathrm{m}$ undercut was realized by the following isotropic plasma dry etching using $\mathrm{CF}_{4} / \mathrm{O}_{2}$ chemistry with a $\mathrm{Si} / \mathrm{SiO}_{2}$ etching selectivity ratio of over 100 .

The scanning electron microscope (SEM) images in Figs. 8(b)-8(d) are beams after undercutting silicon underneath. Most beams bend upward naturally as shown in Fig. 8(b), which was considered as a result of the release of mechanical stress stored in $\mathrm{SiO}_{2}$ during thermal oxidation. In 




FIG. 9. The SEM images of beams (a) before and (b) after being loaded with the tip of the nanoindenter around the center of the beam. The schematic cross-sectional illustrations are shown together. The dashed lines along the edges of the $\mathrm{SiO}_{2}$ beams are the guides to the eyes.

the case of $\mathrm{SiO}_{2}$ formed by plasma enhanced chemical vapor deposition (PECVD), no bent beam was observed as shown in Fig. 8(c). Note that several downward-bent beams were also observed as shown in Fig. 8(d). We also observed various deformation modes in the longer beams. All of these results showed that thermal stress induced during oxidation plays an important role to form initially bent beam, which is a crucial building block for the nonvolatile memory operation in the NEMS memory device. Control of the internal stress in the $\mathrm{SiO}_{2}$ beam is therefore an important issue for further process optimization.

\section{B. Direct observation of mechanical bistability}

In order to investigate the bistable nature of the beam more directly, we applied the nanoindenter type loading system ${ }^{19}$ to our single $\mathrm{SiO}_{2}$ beams. As was already mentioned, most of the fabricated beams bend upward. Figure 9(a) shows a typical SEM image of the upward-bent beam in the test chip. We loaded around the center of the beams with the tip of the nanoindenter. Figure 9(b) shows a SEM image of a single beam taken after loading. We observed clear downward-bent beam, which was the direct observation of the bistable nature of the beam. This result also suggests that the bistable states can be switched by applying an external force. It is important to estimate a value of external force required for switching the beam. No anomaly was, however, observed on the load-displacement curve, which means the force necessary to switch the beam is below $\sim 10 \mu \mathrm{N}$, the resolution limit of the nanoindenter. This result is quantitatively consistent with the calculation results in Fig. 5, where the buckling took place at $\sim 0.5 \mu \mathrm{N}$.

\section{SUMMARY}

We proposed the nanodot NEMS memory device which can realize the high-speed and nonvolatile memory operation and can be fabricated with well-compatible processes with current silicon technologies. Sufficient on-off ratio was estimated from the analysis of the equivalent circuit model. We also suggested that the nc-Si dot incorporation into the movable floating gate beam had the advantage of low power operation. We clearly observed the mechanical bistability of the movable floating gate beam both experimentally and theoretically. About $1 \mathrm{GHz}$ switching speed was estimated by FEM simulation, which is applicable to future high-speed and nonvolatile memory device.

\section{ACKNOWLEDGMENTS}

The authors would like to thank Professor Y. Higo, Professor K. Takashima, and Dr. S. Koyama of Tokyo Institute of Technology for their help in using the nanoindenter apparatus and helpful comments. They would like to thank Dr. T. Arai and Dr. S. Saito of Hitachi Ltd. for fruitful discussion. This work was partly supported by Inter-Research Centers Cooperative Program (IRCP) of the Japan Society for the Promotion of Science.

${ }^{1}$ K. E. Peterson, IBM Tech. Discl. Bull. 20, 5309 (1978).

${ }^{2}$ B. Hälg, IEEE Trans. Electron Devices 37, 2230 (1990).

${ }^{3}$ R. H. Blick et al., J. Phys.: Condens. Matter 14, R905 (2002).

${ }^{4}$ X. M. H. Huang, C. A. Zorman, M. Mehregany, and M. L. Roukes, Nature (London) 421, 496 (2003).

${ }^{5}$ A. N. Cleland and M. L. Roukes, Appl. Phys. Lett. 69, 2653 (1996).

${ }^{6}$ J. Cumings and A. Zettl, Science 289, 602 (2000).

${ }^{7}$ Q. Zheng and Q. Jiang, Phys. Rev. Lett. 88, 045503 (2002).

${ }^{8}$ Y. Tsuchiya et al., IEEE 2004 Silicon Nanoelectronics Workshop, 2004, pp. 101-102.

${ }^{9}$ Y. Tsuchiya, K. Takai, N. Momo, T. Nagami, S. Yamaguchi, T. Shimada, H. Mizuta, and S. Oda, in Physics of Semiconductors: 27th International Conference on the Physics of Semiconductors, edited by J. Menéndez and C. G. V. de Walle (American Institute of Physics, New York, 2005), pp. $1589-1590$.

${ }^{10}$ S. M. Sze, Physics of Semiconductor Devices, 2nd ed. (Wiley, New York, 1981), Chap. 7.

${ }^{11}$ K. Yano et al., Proc. IEEE 87, 633 (1999).

${ }^{12}$ T. Ifuku, M. Otobe, A. Itoh, and S. Oda, Jpn. J. Appl. Phys., Part 2 36, L4031 (1997).

${ }^{13}$ K. Nishiguchi, X. Zhao, and S. Oda, J. Appl. Phys. 92, 2748 (2002).

${ }^{14}$ http://www.j-insight.com/; http://adventure.q.t.u-tokyo.ac.jp/.

${ }^{15}$ M. T. Kim, Thin Solid Films 283, 12 (1996).

${ }^{16}$ L. Gan, B. Ben-Nissan, and A. Ben-David, Thin Solid Films 290-291, 362 (1996).

${ }^{17} \mathrm{http} / / /$ www.nenastran.com/index.php

${ }^{18}$ T. Nagami, H. Mizuta, N. Momo, Y. Tsuchiya, S. Saito, T. Arai, T. Shimada, and S. Oda (unpublished).

${ }^{19}$ K. Takashima, Y. Higo, S. Sugiura, and M. Shimojo, Mater. Trans. 42, 68 (2001). 